\title{
THE NUMBER OF ROOTS IN A SIMPLY-CONNECTED $H$-MANIFOLD
}

BY

\author{
ROBERT F. BROWN(1) AND RONALD J. STERN(2)
}

ABSTRACT. An $H$-manifold is a triple $(M, m, e)$ where $M$ is a compact connected triangulable manifold without boundary, $e \in M$, and $m: M \times M \rightarrow M$ is a map such that $m(x, e)=m(e, x)=x$ for all $x \in M$. Define $m_{1}: M \rightarrow M$ to be the identity map and, for $k \geq 2$, define $m_{k}: M \rightarrow M$ by $m_{k}(x)=m\left(x, m_{k-1}(x)\right)$. It is proven that if $(M, m, e)$ is an $H$-manifold, then $M$ is simply-connected if and only if given $k \geq 1$ there exists a multiplication $m^{\prime}$ on $M$ homotopic to $m$ such that $m_{j}^{\prime}(x)=e$ implies $x=e$ for all $j \leq k$.

Throughout this paper, manifold will mean a compact connected triangulable manifold without boundary. We define an $H$-manifold to be a triple $(M, m, e)$ where $M$ is a manifold, $e$ is a point of $M$, and $m: M \times M \rightarrow M$ is a map such that $m(x, e)$ $=m(e, x)=x$ for all $x$ in $M$. The map $m$ is called a multiplication on $M$ with unit $e$. Two such multiplications $m$ and $m^{\prime}$ are bomotopic if there is a continuous family $m(t)$ of multiplications on $M$ with unit $e$, for $t \in I$, such that $m(0)=$ $m$ and $m(1)=m^{\prime}$.

Given an $H$-manifold $(M, m, e)$, define $m_{1}: M \rightarrow M$ to be the identity map and, for $k \geq 2$, define $m_{k}: M \rightarrow M$ by $m_{k}(x)=m\left(x, m_{k-1}(x)\right)$. If $m_{k}(x)=y$, then $x$ is a $k$ th root of $y$.

Hopf [5] observed that every element in a compact connected Lie group has at least $k^{\lambda} k$ th roots; where $\lambda$ is the rank of the Lie group. In contrast to Hopf's result, it was proved in [4] that, given $k \geq 2$ and an $H$-manifold $\left(s^{3}, m, 1\right)$, there is a multiplication $m^{\prime}$ on $S^{3}$ homotopic to $m$ such that $m_{j}^{\prime}(x)=1$ only if $x=1$; for all $j \leq k$. The argument in [4] makes essential use of the fact that $S^{3}$ is a suspension. The purpose of this paper is to extend that result to all simply-connected $H$-manifolds.

Theorem. Let $(M, m, e)$ be an $H$-manifold. The manifold $M$ is simply-connected if and only if given $k \geq 1$ there exists a multiplication $m^{\prime}$ on $M$ bomotopic to $m$ such that $m_{j}^{\prime}(x)=e$ implies $x=e$; for all $j \leq k$.

The proof of sufficiency is already in the literature. Let $\pi$ denote the fundamental group of $M$ and define

Presented to the Society, J anuary 17, 1972; received by the editors September 22, 1971. AMS 1970 subject classifications. Primary 55D45.

Key words and phrases. $H$-space, $k$ th root, coincidence-preserving homotopy.

(1) Research supported in part by NSF Grant GP-29639.

(2) Research supported in part through an NSF traineeship. 


$$
k \pi=\{\alpha \in \pi \mid \alpha=k \beta \text { for some } \beta \in \pi\} .
$$

The proof of the theorem in [2] shows that, for any multiplication on $M$, the number of $k$ th roots of any point is at least as large as the order of the group $\pi / k \pi$.

The rest of this paper will be devoted to the proof of necessity. A special case of the result-when $k$ is no greater than three-was presented by the first author to the Second Florida Symposium on Automata and Semigroups in April 1971. He takes this opportunity to thank Professor A. D. Wallace for the invitation to take part in the Symposium and also the University of Florida for its support and hospitality.

Denote euclidean space by $R^{n}$, the origin in $R^{n}$ by 0 , and the distance from $x \in R^{n}$ to the origin by $|x|$. For $r>0$, let

$$
r B^{n}=\left\{x \in R^{n}|| x \mid<r\right\}
$$

but write $B^{n}$ for $1 B^{n}$. We use $\partial S$ and $\bar{S}$ to denote the boundary and closure of a set $S$.

By imbedding a manifold $M$ into $R^{N}$ for large enough $N$ we immediately get

Lemma 1. Let $M$ be a manifold of dimension $n$ with metric $d^{\prime}$. Let $e \in M$ be a given point. Then there is a neighborbood $U$ of $e$ and an equivalent metric $d$ on $M$ such that $U$ is isometric with an open subset of $R^{n}$ containing the origin.

Lemma 2. Suppose $f_{1}$ and $f_{2}$ are maps from an open subset $U$ of $R^{n}$, containing $2 B^{n}$, into $R^{n}$ such that $f_{1}^{-1}(0)=f_{2}^{-1}(0)=0$. Then there exists a map $g: U \rightarrow R^{n}$ sucb that

(i) $g^{-1}(0)=0$,

(ii) $g=f_{2}$ outside of $2 B^{n}$,

(iii) $\left|f_{1}(x)\right|<|g(x)|$ for $x \in \overline{B^{n}}-\{0\}$.

Proof. Define $g(0)=0$ and $g(x)=f_{2}(x)$ if $|x| \geq 2$. Otherwise, let

where

$$
\begin{array}{rlrl}
g(x) & =\left(\left(\left|f_{1}(x)\right|+|x|\right) /\left|f_{2}(x)\right|\right) f_{2}(x) & & \text { if } 0<|x| \leq 1, \\
& =\left(a(x) /\left|f_{2}(x)\right|\right) f_{2}(x) & \text { if } 1 \leq|x| \leq 2
\end{array}
$$

$$
a(x)=\left(\left|f_{2}(x)\right|-\left|f_{1}(x)\right|-1\right)|x|+2\left|f_{1}(x)\right|+2-\left|f_{2}(x)\right| .
$$

For maps $f, g: X \rightarrow Y$ define

$$
C(f, g)=\{x \in X \mid f(x)=g(x)\} .
$$

A homotopy $H: X \times I \rightarrow Y$ between $f$ and $g$ will be called coincidence-preserving if $H(x, t)=f(x)$ for all $t$ whenever $x \in C(f, g)$.

Lemma 3. Let $M$ be a manifold and $e \in M$. Let $U$ and $d$ be as in Lemma 1. 
Suppose $f_{1}, f_{2}: M \rightarrow M$ are maps such that $f_{1}^{-1}(e)=f_{2}^{-1}(e)=e$. Suppose $\epsilon>0$ is small

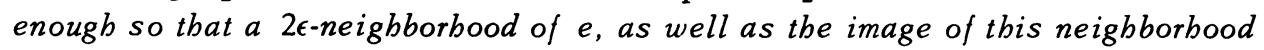
under $f_{1}$ and $f_{2}$, is contained in $U$. Then if $d\left(f_{1}(x), e\right) \geq d(x, e)$ when $d(x, e) \leq \epsilon$, there is a map $g: M \rightarrow M$ such that

(i) $g^{-1}(e)=e$,

(ii) $d\left(f_{1}(x), e\right)<d(g(x), e)$ if $0<d(x, e) \leq \epsilon$,

(iii) $d(g(x), e) \geq \epsilon$ if $d(x, e) \geq \epsilon$,

(iv) if $d(x, e) \geq 2 \epsilon$ and $d\left(f_{2}(x), e\right)>\epsilon$, then $f_{2}(x)=g(x)$,

(v) $g$ is coincidence-preserving bomotopic to $f_{2}$.

Proof. Lemmas 1 and 2 give a map $g^{\prime}: M \rightarrow M$ satisfying all the conclusions of the lemma except, perhaps, conclusions (iii) and (iv). Let $\rho: M-\{e\} \rightarrow M$ be the map induced by the radial projection of $\overline{B^{n}}-\{0\}$ onto $\partial B^{n}$. Define $g(x)=$ $g^{\prime}(x)$ if $d(x, e) \leq \epsilon$ and $g(x)=\rho g^{\prime}(x)$ if $d(x, e) \geq \epsilon$.

Lemma 4. Let $M$ be a manifold with metric $d$ and let $x_{0} \in M$. Suppose that $\epsilon>0$ is less than one and that $f, g, a_{1}, \cdots, a_{q}$ are maps from an open subset $U$ of $M$, containing $\left\{x \in M \mid d\left(x, x_{0}\right)<2 \epsilon\right\}$ into $R^{n}$, such that

(a) $f\left(x_{0}\right)=g\left(x_{0}\right)=a_{i}\left(x_{0}\right)$ for $1 \leq i \leq q$,

(b) $f(x) \neq g(x)$ and $g(x) \neq a_{i}(x)$ for $1 \leq i \leq q$ when $\theta<d\left(x, x_{0}\right)<2 \epsilon$.

Then there exists a map $f^{\prime}: U \rightarrow R^{n}$ such that

(i) $f^{\prime}\left(x_{0}\right)=g\left(x_{0}\right)$,

(ii) $f^{\prime}(x)=f(x)$ when $d\left(x, x_{0}\right) \geq 2 \epsilon$,

(iii) $f^{\prime}(x) \neq g(x)$ when $0<d\left(x, x_{0}\right)<2 \epsilon$,

(iv) $\left|f^{\prime}(x)-g(x)\right|<\Pi_{i=1}^{q}\left|g(x)-a_{i}(x)\right|$ when $0<d\left(x, x_{0}\right)<\epsilon$,

(v) $f^{\prime}$ is coincidence-preserving bomotopic to $f$.

Proof. When $0<d\left(x, x_{0}\right)<2 \epsilon$, let

$$
\beta(x)=\left(\prod_{i=1}^{q}\left|g(x)-a_{i}(x)\right|\right) /|g(x)-f(x)| .
$$

Define $f^{\prime}: U \rightarrow R^{n}$ by setting $f^{\prime}\left(x_{0}\right)=g\left(x_{0}\right), f^{\prime}(x)=f(x)$ for $d\left(x_{0} x_{0}\right) \geq 2 \epsilon$ and

$$
\begin{aligned}
f^{\prime}(x) & =\left[\left(\epsilon-\epsilon d\left(x, x_{0}\right)+\left(d\left(x, x_{0}\right)\right)^{2}\right) \beta(x)\right](f(x)-g(x))+g(x) \quad \text { if } 0<d\left(x, x_{0}\right) \leq \epsilon, \\
& =\left[((1-\epsilon \beta(x)) / \epsilon) d\left(x, x_{0}\right)+2 \epsilon \beta(x)-1\right](f(x)-g(x))+g(x) \\
& \text { if } \epsilon \leq d\left(x, x_{0}\right) \leq 2 \epsilon .
\end{aligned}
$$

Straightforward modifications of the proof of Theorem 2 of [6] produce the following result:

Lemma 5. Let $M$ be a manifold and let $N$ denote a neighborbood of $e \in M$. Let f. $g: M \rightarrow M$ be maps taking $M-N$ to itself sucb that $C(f, g) \cap \bar{N}=\{e\}$. Suppose 
there are given finite subsets $A, B$ and $C$ of $M-\vec{N}$, with $A \cap B=\emptyset$, and $\epsilon>0$, smaller than the distance from $C(f, g) \cup A \cup B$ to $\partial N$, baving the property that for any point $x_{0} \in C$ a $2 \epsilon$-neighborbood $N\left(x_{0}\right)$ of $x_{0}$ is in a euclidean neigbborbood of $M-N$ and $N\left(x_{0}\right) \cap C(f, g)=\left\{x_{0}\right\}$. There exists a map $f^{\prime}: M \rightarrow M$ sucb that

(i) $d\left(f, f^{\prime}\right)<\epsilon$,

(ii) $f^{\prime}=f$ outside of a $2 \epsilon$-neighborbood of $C(f, g)-C$,

(iii) $C\left(f^{\prime}, g\right)$ is finite,

(iv) $\left[C\left(f^{\prime}, g\right)-C\right] \cap A=\varnothing$,

(v) $f^{\prime}=f$ on $B$.

The next result is an easy application of the Homotopy Extension Theorem (compare [2, p. 186]).

Lemma 6. Let $(M, m, e)$ be an H-manifold, let $P$ and $Q$ be closed subsets of $M \times M$, and let $H: Q \times I \rightarrow M$ be a map such that $H(x, y, t)=m(x, y)$ whenever $(x, y) \in P \cap Q, x=e$ or $y=e$. Then there exists a multiplication $m^{\prime}$ on $M$ bomo. topic to $m$ such that $m^{\prime}=m$ on $P$ and $m^{\prime}(x, y)=H(x, y, 1)$ for $(x, y) \in Q$.

Another application of the Homotopy Extension Theorem gives us

Lemma 7. Let $M$ be a simply-connected manifold, let $f, g: M \rightarrow M$ be bomotopic maps, and let $S$ be a finite subset of $M$. Then there is a map $g^{\prime}: M \rightarrow M$ identical to $g$ outside of an arbitrarily small neighborbood of $S$ and bomotopic to $f$ by a bomotopy $H$ with the property $H(x, t)=f(x)$ for all $x \in S$.

We have now collected all the constructions we need in order to prove the theorem. We make the following induction assumptions:

$$
\begin{aligned}
& (*)_{k} \quad m_{k}(x)=e \text { implies } x=e \text {, } \\
& (* *)_{k-1} \quad \text { there exists } \epsilon>0 \text { sucb that } \\
& \text { (a) } d(x, e)<d\left(m_{2}(x), e\right)<\cdots<d\left(m_{k-1}(x), e\right) \text { if } 0<d(x, e) \leq \epsilon \text {, } \\
& \text { (b) } d\left(m_{j}(x), e\right) \geq \epsilon \text { if } d(x, e) \geq \epsilon \text { and } j \leq k-1, \\
& (* * *)_{k-1} C\left(m_{i}, m_{j}\right) \text { is finite if } 1 \leq i<j \leq k-1 .
\end{aligned}
$$

The strategy of the proof is to modify the multiplication $m$ through homotopies so that $(* *)_{k}$ is satisfied, then in addition $(* * *)_{k}$ is satisfied, and finally $(*)_{k+1}$ is satisfied as well.

Step $(* *)_{k}$. Applying Lemma 3 with $f_{1}=m_{k-1}$ and $f_{2}=m_{k}$, there is a map $g: M \rightarrow M$ homotopic to $m_{k}$ such that $d\left(m_{k-1}(x), e\right)<d(g(x), e)$ when $d(x, e) \leq \epsilon$. Let $B=\bigcup_{i<k-1} C\left(m_{i}, m_{k-1}\right)-\{e\}$. Taking $\epsilon$ in Lemma 3 small enough so that $d(x, e)>2 \epsilon$ and $d\left(m_{k-1}(x), e\right)>\epsilon$ for all $x \in B$, we know that $g$ has the additional property that $g=m_{k}$ on $B$ and that the homotopy is coincidence-preserving on $B$. Let 


$$
\partial_{i}=\left\{\left(x, m_{i}(x)\right) \in M \times M \mid x \in M\right\}
$$

and apply Lemma 6 for $P=\bigcup_{i=1}^{k-2} \partial_{i}, Q=\partial_{k-1}$ and the homotopy induced by that between $m_{k}$ and $g$. We obtain a multiplication $m^{\prime}$ on $M$ with $m_{i}^{\prime}=m_{i}$ for $i \leq k-1$ and $m_{k}^{\prime}=g$.

Step $(* * *)_{k}$. We assume that $m$ is a multiplication on $M$ satisfying $(*)_{k}$, $(* *)_{k}$ and $(* * *)_{k-1}$. Let $\omega>0$ be chosen so that if $f, g: M \rightarrow M$ are maps where $d(f, g)<\omega$, then $f$ and $g$ are homotopic by a coincidence-preserving homotopy [3, pp. 39-40]. Let $B$ be defined as above. By Lemma 5, there exists a map $f: M \rightarrow M$ such that $d\left(f, m_{k}\right)<\omega, C\left(m_{1}, f\right)$ is finite, and $f(x)=m_{k}(x)$ if $x \in B$ or if $d(x, e) \leq \epsilon$. Applying Lemma 6 produces a multiplication $m^{\prime}$ on $M$ such that $m_{i}^{\prime}=m_{i}$ for $1 \leq i \leq k-1$ and $m_{k}^{\prime}=f$ so $m^{\prime}$ has the same properties as $m$ and, in addition, $C\left(m_{1}^{\prime}, m_{k}^{\prime}\right)$ is finite.

We now further assume that for some $1 \leq r \leq k-1$ we have

$(* * *)_{k}(r) \quad C\left(m_{i}, m_{k}\right)$ is finite for $1 \leq i \leq r-1$.

We treated the case $(* * *)_{k}(2)$ in the previous paragraph.. Since $(* * *)_{k}(k)=$ $(* * *)_{k}$, it remains to establish the induction step.

First set up the following notation:

$$
\begin{aligned}
A & =\left[\bigcup_{i<r} C\left(m_{i}, m_{k}\right) \cup \underset{i<j<r}{\bigcup} C\left(m_{i}, m_{j}\right)\right]-B-\{e\}, \\
D & =\left\{x \in M-\{e\} \mid m_{i}(x)=m_{r}(x)=m_{k}(x) \text { for some } 1 \leq i \leq r-1\right\}, \\
\partial_{i j} & =\left\{(x, y) \in M \times M-\{(e, e)\} \mid m_{i}(x)=m_{j}(x)=y\right\}, \\
\Delta & =\left\{(x, y) \in M \times M \mid x \in D \text { and } y=m_{k-1}(x)\right\} .
\end{aligned}
$$

On $M \times M$ we use the metric $d^{\prime}$ that is defined by

$$
d^{\prime}\left[\left(x_{1}, y_{1}\right),\left(x_{2}, y_{2}\right)\right]=\max \left[d\left(x_{1}, x_{2}\right), d\left(y_{1}, y_{2}\right)\right] \text {. }
$$

Let $W$ be a $\gamma^{\prime}$-neighborhood of $\partial_{r k}$ in $M \times M$; where $\gamma^{\prime}$ is chosen small enough so that

$$
(W-\Delta) \cap \bigcup_{i<j<r} \partial_{i j}=\varnothing .
$$

There exists $\gamma, 0<\gamma<\gamma^{\prime}$, such that $d(x, y)<\gamma$ implies $d\left(m_{k}(x), m_{k}(y)\right)<$ $\gamma^{\prime} / 2$. Let $\tau$ be the Lebesgue number of a finite cover of $M$ by euclidean neighborhoods and define $\eta$ by letting $2 \eta$ be the smallest of $\epsilon$ (from step $\left.(* *)_{k}\right), \omega, \gamma$ and $\tau$.

By Lemma 5 , there is a map $f: M \rightarrow M$ with the following properties:

(i) $d\left(f, m_{k}\right)<\eta$,

(ii) $f=m_{k}$ outside of a $2 \eta$-neighborhood $U$ of $C\left(m_{r}, m_{k}\right)$,

(iii) $C\left(f, m_{r}\right)$ is finite,

(iv) $C\left(f, m_{r}\right) \cap A=\varnothing$,

(v) $f=m_{k}$ on $B$. 
Let

$$
C=\left\{x \in M-\{e\} \mid f(x)=m_{r}(x)=m_{i}(x) \text { for some } i<r\right\} \text {. }
$$

Lemma 8. If $f\left(x_{0}\right)=m_{i}\left(x_{0}\right)=m_{j}\left(x_{0}\right)$ for some $i<j<r$ and $x_{0} \in \bar{U}$, then $x_{0} \in C$.

Proof. Let $(x, y) \in \partial_{r k}$ be chosen so that $d\left(x_{0}, x\right)<2 \eta$, then $d^{\top}\left[\left(x_{0}, f\left(x_{0}\right)\right),(x, y)\right]$ $<\gamma^{\prime}$. Thus $\left(x_{0}, f\left(x_{0}\right)\right) \in W$ and, by hypothesis, $\left(x_{0}, f\left(x_{0}\right)\right) \in \partial_{i j}$ so it must be that $\left(x_{0}, f\left(x_{0}\right)\right) \in \Delta$ since $(W-\Delta) \cap \partial_{i j}=\varnothing$. Consequently, $f\left(x_{0}\right)=m_{k}\left(x_{0}\right)=$ $m_{r}\left(x_{0}\right)$.

For $p \in C$, we define $\mathcal{P}$ to be the set of those integers between one and $r-1$ for which $m_{i}(p)=m_{r}(p)=f(p)$ and $\widetilde{P}$ to be the complement of $\mathcal{P}$ in $\{1,2$, $\cdots, r-1\}$. Let $\beta>0$ be such that, for $N(p)$ a $\beta$ neighborhood of $p$ and $N^{0}(p)=$ $N(p)-\{p\}, f\left(\overline{N(p))}\right.$ and $\left.m_{i} \overline{(N(p)}\right), 1 \leq i \leq r$, are contained in a euclidean neighborhood $V$ of $f(p)$. Let $b: V \rightarrow R^{n}$ be a homeomorphism such that $b(N(p))$ is contained in a ball of radius less than one about $b(p)$. We also require that $\beta$ satisfy

(i) $B \cap N^{0}(p)=\varnothing$,

(ii) $f(x) \neq m_{r}(x)$ for $x \in N^{0}(p)$,

(iii) $m_{r}(x) \neq m_{i}(x)$ for $i \in \mathcal{P}$ and $x \in N^{0}(p)$,

(iv) there exists $0<a<\beta / 2$ such that $\left|b f(x)-b m_{i}(x)\right|>\alpha$ where $i \in \mathfrak{P}$ and $x \in \overline{N(p)}$

(v) $\left|b m_{i}(x)-b m_{i}(y)\right|<\alpha / 4$ for $x, y \in \overline{N(p)}$ and either $i \in \mathcal{P}$ or $i=r$.

By Lemma 4 , there exists a map $f^{\prime}: N^{0}(p) \rightarrow R^{n}$ homotopic to $\left.b f\right|_{N(p)}$ such that $f^{\prime}\left(p_{0}\right)=b f\left(p_{0}\right)$,

$$
\left|f^{\prime}(x)-b m_{r}(x)\right|<\prod_{i \in \Phi}\left|b m_{i}(x)-b m_{r}(x)\right|
$$

when $d(x, p)<\beta / 2, f^{\prime}(x)=b f(x)$ if $d(x, p) \geq \beta$ and $f^{\prime}(x) \neq b m_{r}(x)$ if $0<d(x, p)<$ $\beta$. If $f^{\prime}\left(x_{0}\right)=b m_{j}\left(x_{0}\right)$ for some $x_{0}$ where $0<d\left(x_{0}, p\right)<\beta / 2$ and some $j \in \mathcal{P}$, then we would have

$$
\prod_{i \in \boldsymbol{\Phi}_{-}\{j\}}\left|b m_{i}\left(x_{0}\right)-b m_{r}\left(x_{0}\right)\right|>1
$$

which is impossible because $\left|b m_{i}\left(x_{0}\right)-b m_{r}\left(x_{0}\right)\right|<\alpha / 2$ for all $i \in \mathcal{P}$. If $f^{\prime}\left(x_{0}\right)=$ $b m_{i}\left(x_{0}\right)$ for some $x_{0}$ where $0<d\left(x_{0}, p\right)<\beta / 2$ and some $i \in \widetilde{P}$, then $\mid b f\left(x_{0}\right)-$ $b m_{i}\left(x_{0}\right) \mid<\alpha$, contrary to the definition of $\alpha$. Thus, by considering $b^{-1} f^{\prime}$ we may assume without loss of generality that the map $f$ homotopic to $m_{k}$ has the additional properties that for each $p \in C$ there is a neighborhood $N(p)$ for which

$$
\begin{aligned}
C\left(f, m_{i}\right) \cap N(p) & =\{p\} & & \text { if } i \in \mathscr{P}, \\
& =\varnothing & & \text { if } i \in \widetilde{\mathscr{P}} .
\end{aligned}
$$


Let $s$ be the smallest integer between one and $r-1$ such that $C\left(f, m_{s}\right)$ is infinite. We claim that there exists $\sigma>0$ such that if $x_{0}$ is within $\sigma$ of $C\left(f, m_{s}\right)$ $-C$ then $f\left(x_{0}\right) \neq m_{i}\left(x_{0}\right)$ for all $i \neq s^{\prime}$ between one and $r-1$. To verify the claim, note that if $x_{0}$ is in both $C\left(f, m_{s}\right)$ and $C\left(f, m_{i}\right)$, then $x_{0} \in C$ by Lemma 8 . The previous paragraph established that $f$ can be assured to have the property that the elements of $C$ are isolated points of $C\left(f, m_{s}\right)$. Thus $C\left(f, m_{s}\right)-C$ and $C\left(f, m_{i}\right)$ are disjoint compact sets when $i \neq s$, and therefore $\sigma$ exists. Let $V$ denote the closure of a $\sigma$-neighborhood of $C\left(f, m_{s}\right)-C$ and let

$$
\delta_{i}=\inf \left\{d\left(f(x), m_{i}(x)\right) \mid x \in V\right\}>0 .
$$

Choose $\delta>0$ to be smaller than $\sigma$ and all the $\delta_{i}$.

By Lemma 5, there exists $f^{\prime}: M \rightarrow M$ such that

(i) $d\left(f, f^{\prime}\right)<\delta / 2$,

(ii) $f^{\prime}=f$ outside of $V$,

(iii) $C\left(f^{\prime}, m_{s}\right)$ is finite,

(iv) $f^{\prime}=f$ on $B$.

The definition of $\delta$ guarantees that $C\left(f^{\prime}, m_{i}\right)=C\left(f, m_{i}\right)$ for all $i \neq s$. Thus, in a finite number of steps we obtain a map $f^{\prime}$ homotopic to $m_{k}$ such that $C\left(f, m_{i}\right)$ is finite for all $i=1,2, \cdots, r$. Furthermore, if $m_{k-1}\left(x_{0}\right)=m_{i}\left(x_{0}\right)$, in other words if $x_{0}$ is in $B$, then $f^{\prime}\left(x_{0}\right)=m_{k}\left(x_{0}\right)$ and the homotopy is coincidence-preserving at that point. Applying Lemma 6 to the homotopy between $m_{k}$ and $f^{\prime}$ we obtain a multiplication $m^{\prime}$ on $M$ homotopic to $m$ such that $m_{i}^{\prime}=m_{i}$ for $1 \leq i \leq k-1$ and $m_{k}^{\prime}=f^{\prime}$. Consequently, $C\left(m_{i}^{\prime}, m_{k}^{\prime}\right)=C\left(m_{i}, f^{\prime}\right)$ is finite for $i=1,2, \cdots, r$.

Step $(*)_{k+1}$. We now assume that the multiplication $m$ on $M$ satisfies $(*)_{k}$, $(* *)_{k}$ and $(* * *)_{k}$. By Proposition 4 of [1], since $M$ is simply-connected there is a map $g: M \rightarrow M$ homotopic to $m_{k+1}$ such that $g^{-1}(e)=e$. By Lemma 7, we may assume that $g=m_{k+1}$ on $\bigcup_{i<k} C\left(m_{i}, m_{k}\right)$ and that the homotopy is coincidencepreserving on that set. The application of Lemma 6 completes the proof.

\section{REFERENCES}

1. R. Brooks, On removing coincidences of two maps when one, rather than both, of them may be deformed by a homotopy, Pacific J. Math. 40 (1972), 45-52.

2. R. F. Brown, Divisible H-spaces, Proc. Amer. Math. Soc 26 (1970), 185-189. MR $41 \# 6207$.

3. - The Lefschetz fixed point theorem, Scott, Foresman, Glenview, Ill. 1971.

4. R. F. Brown and A. Hales, Primitive roots of unity in $\mathrm{H}$-manifolds, Amer. J. Math. $92(1970), 612-618$.

5. H. Hopf, Über den Rang geschlossener Liescher Gruppen, Comment. Math. Helv. 13 (1940), 119-143. MR 3, 35.

6. H. Schirmer, Mindestzahlen von Koinzidenzpunkten, J. Reine Angew. Math. 194 (1955), 21-39. MR 17, 394.

DEPARTMENT OF MATHEMATICS, UNIVERSITY OF CALIFORNIA, LOS ANGELES, CALIFORNIA 90024 